\title{
Naturalness and light higgsinos: A powerful reason to build the ILC
}

\author{
Howard Baer* \\ Univesity of Oklahoma, Norman, OK 73019, USA \\ E-mail: baereou.edu \\ Jenny List ${ }^{\dagger}$ \\ DESY, 22603 Hamburg, Germany \\ E-mail: jenny.listedesy.de
}

Mikael Berggren, Suvi-Leena Lehtinen

DESY, 22603 Hamburg, Germany

E-mail: mikael.berggren@desy.de, suvi-leena.lehtinen@desy.de

\section{Keisuke Fujii, Jacqueline Yan}

KEK, Tsukuba, Japan

E-mail: keisuke.fujii@kek.jp, jackie@post.kek.jp

\section{Tomohiko Tanabe}

International Center for Elementary Particle Physics, University of Tokyo, Tokyo, Japan

E-mail: tomohiko@icepp.s.u-tokyo.ac.jp

\begin{abstract}
A core prediction of natural Supersymmetry is the existence of four light higgsinos not too far above the mass of the $Z$ boson. The small mass splittings amongst the higgsinos - typically 5$20 \mathrm{GeV}$ - imply very little visible energy release from decays of heavier higgsinos. In particular, if other SUSY particles are quite heavy, as can be the case in SUSY with radiatively-driven naturalness, the higgsinos are extremely hard to detect at hadron colliders. The clean environment of electron-positron colliders with $\sqrt{s}>2 m_{\text {higgsino, }}$, however, would allow for a decisive search for the required light higgsinos. Thus, $e^{+} e^{-}$colliders should either discover or exclude natural SUSY. We present a detailed study of higgsino pair production at the proposed International Linear $e^{+} e^{-}$Collider which is under consideration for construction in Japan. A variety of precision measurements should allow for extraction of underlying parameters and provide a window onto physics at the grand unified scale.
\end{abstract}

38th International Conference on High Energy Physics

3-10 August 2016

Chicago, USA

\footnotetext{
*Speaker.

† Speaker.
} 


\section{Motivation}

In thinking about physics Beyond the Standard Model (BSM), especially when it comes to motivation for constructing costly new facilities, it pays to bear in mind two gems of wisdom:

- Everything should be made as simple as possible, but not simpler. (A. Einstein)

- The appearance of fine-tuning in a scientific theory is like a cry of distress from nature, complaining that something needs to be better explained. (S. Weinberg)

The first of these we interpret as meaning: the further one strays from the Standard Model (SM), the more likely one is to be wrong. The second we interpret as meaning: nature is natural so any theory depending on an unexplained fine-tuning is likely a wrong theory.

Now, whereas the SM provides an excellent description of a vast panoply of data, it is known to be highly unnatural in the scalar (Higgs) sector if its regime of validity as an effective theory is taken much beyond the $\Lambda \sim 1 \mathrm{TeV}$ energy scale. In years past, this fact had led some theorists to question whether fundamental scalar fields could exist in nature. Many solutions to the SM naturalness problem have been invoked, but only one appears consistent with data and with the above two missives: extending the underlying spacetime symmetry group to its most general structure: the super-Poincaré algebra, or supersymmetry (SUSY)[1]. Under SUSY, quadratic divergences neatly cancel to all orders in perturbation theory, rendering the theory natural. It may even be claimed that SUSY theories have generated three predictions found to be in accord with data: 1 . the measured relative strengths of the weak scale gauge couplings, 2 . the value of the top quark mass is just what is needed to generate a radiative breakdown of electroweak symmetry and 3. the measured value of the new-found Higgs boson mass $m_{h}=125.1 \mathrm{GeV}[2,3]$ lies squarely within the narrow window predicted by the Minimal Supersymmetric Standard Model (MSSM)[4].

Alas, a fourth prediction, the existence of superpartner matter states, has yet to be verified. Recent searches at LHC with $\sqrt{s}=13 \mathrm{TeV}$ find, within the context of various simplified models, that $m_{\tilde{g}} \gtrsim 1.9 \mathrm{TeV}[5]$ and $m_{\tilde{t}_{1}} \gtrsim 0.85 \mathrm{TeV}[6]$. In addition, the measured value of $m_{h}=125.1 \mathrm{GeV}$ seems to require highly mixed TeV-scale top-squarks[7]. These various mass bounds are in deep discord from early naturalness constraints, this time arising from logarithmic rather than quadratic divergences, that $m_{\tilde{g}} \lesssim 350 \mathrm{GeV}[8]$ and that $m_{\tilde{t}_{1}} \lesssim 350 \mathrm{GeV}$ [9] (for fine-tuning measure $\Delta_{B G}<30$ ). This discord has led many physicists to question whether weak scale SUSY is indeed the path forward after all[10]. It has also led to renewed scrutiny as to the validity of the earlier naturalness estimates[11, 12, 13, 14, 15].

The most direct relation between the observed value of the weak scale and elements of the SUSY Lagrangian comes from minimizing the MSSM scalar potential. Then it is found that

$$
\frac{m_{Z}^{2}}{2}=\frac{m_{H_{d}}^{2}+\Sigma_{d}^{d}-\left(m_{H_{u}}^{2}+\Sigma_{u}^{u}\right) \tan ^{2} \beta}{\tan ^{2} \beta-1}-\mu^{2} \simeq-m_{H_{u}}^{2}-\Sigma_{u}^{u}-\mu^{2}
$$

where $\tan \beta \equiv v_{u} / v_{d}$ is the ratio of Higgs field vacuum expectation values, $m_{H_{u}}^{2}$ and $m_{H_{d}}^{2}$ are Higgs field soft SUSY breaking terms and $\mu$ is the superpotential Higgs/higgsino mass term. If any term on the right-hand-side is far greater than $m_{Z}^{2} / 2$, then some other (completely unrelated) term would have to be (implausibly) fine-tuned to an opposite-sign value such as to maintain $m_{Z}$ at its measured 
value. Alternatively, for a natural theory, all terms on the RHS should be comparable to or smaller than $m_{Z}^{2} / 2$. The electroweak fine-tuning measure $\Delta_{E W}$ was introduced[11, 12] to measure the largest contribution on the RHS of Eq. 1.1 compared to $m_{Z}^{2} / 2$. Then, for a natural (i.e. plausible) theory with low $\Delta_{E W}$, one finds the following. 1 . The soft parameter $m_{H_{u}}^{2}$ is driven radiatively to small negative values at the weak scale. 2 . In order that the radiative corrections $\Sigma_{u}^{u} \lesssim m_{Z}^{2} / 2$, the top-squarks are highly mixed (large $A_{t}$ ) and not too far beyond the few $\mathrm{TeV}$ range. This last requirement is completely consistent with the measured value of $m_{h}$ and with expectations from the measured value of $B F(b \rightarrow s \gamma)$. In addition, the gluino mass feeds into the $\Sigma$ terms and thus $m_{\tilde{g}} \lesssim 4 \mathrm{TeV}$ (perhaps well beyond the reach of even HL-LHC)[12, 16]. 3. Finally, $\mu \sim 100-300$ $\mathrm{GeV}$, the closer to $m_{Z}$ the better.

This latter condition implies the existence of four higgsino-like states $\tilde{\chi}_{1}^{ \pm}$and $\tilde{\chi}_{1,2}^{0}$ with mass $\sim 100-300 \mathrm{GeV}$ (the closer to $m_{Z}$ the better). These are the only SUSY particles required to be near the weak scale. Furthermore, in spite of their light mass, they are very difficult to detect at LHC due to their compressed spectra: the lightest higgsino-like electroweakino (EWino) is typically just 5-20 GeV lighter than the heavier ones. Since $\tilde{\chi}_{1}^{0}$ would constitute a portion of the dark matter (along with perhaps axions[17]), the bulk of energy produced from higgsino decays becomes bound up in the $\tilde{\chi}_{1}^{0}$ mass: thus, the lightest state is totally invisible while the heavier states lead only to soft particles which are difficult to distinguish from QCD backgrounds.

On the other hand, these light higgsinos would be easily visible at an $e^{+} e^{-}$collider such as

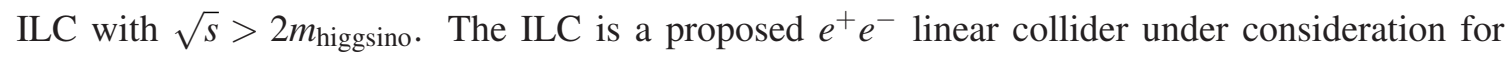
construction in Japan. Just above kinematic threshold, the higgsino pair production cross sections lie in the $10^{2}-10^{3} \mathrm{fb}$ range. Such a machine, intended to be a Higgs factory, would turn out to be as well a higgsino factory[18]!

Overall, low values of $\Delta_{E W} \lesssim 30^{1}$ allow for a Little Hierarchy (LH) $\mu \ll m_{S U S Y}$, but such a LH is not a problem since $m_{S U S Y}$ enters the weak scale via radiative corrections while $\mu$ enters the weak scale at tree level.

What of the earlier naturalness estimates? The BG measure $\Delta_{B G} \equiv \max _{i}\left|\frac{\partial \ln m_{Z}^{2}}{\partial p_{i}}\right|$ (where $i$ labels fundamental parameter $p_{i}$ ) was typically evaluated in terms of multi-soft-parameter effective theories. In more fundamental theories where the soft terms are all derived in terms of say the more fundamental gravitino mass $m_{3 / 2}$, then $\Delta_{B G}$ reduces to $\Delta_{E W}[14]$. Other evaluations of logarithmic corrections to $m_{H_{u}}^{2}$ compared to $m_{h}^{2}$ [19] were found to neglect the $m_{H_{u}}^{2}$ self-contribution to RG running[13]. By including this, one allows for radiatvely-driven naturalness (RNS) where the large top-Yukawa coupling and large soft terms drive $m_{H_{u}}^{2}$ from large, unnatural high scale values to natural values at the weak scale.

\section{Natural SUSY at the ILC: From masses and cross sections to SUSY parameters}

The prospects for mass and cross section measurements have been studied in a full, GEANT4based [20] simulation of the ILD detector concept proposed for the ILC, using as an example the NUHM2 benchmark ILC1 [18]. Collision events were generated with WHIZARD 1.95 [21] including beamstrahlung and ISR, as well as hadronisation by PYTHIA 6.422 [22] tuned to LEP data.

\footnotetext{
${ }^{1}$ The onset of fine-tuning for $\Delta_{E W} \gtrsim 30$ is visually displayed in Ref. [16].
} 

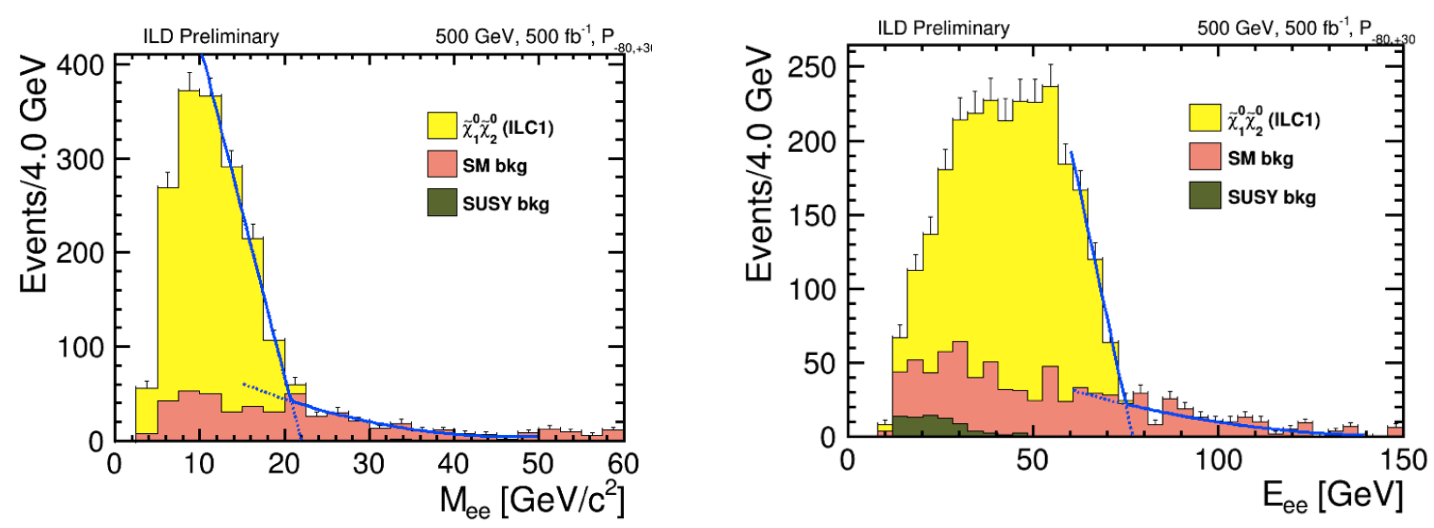

Figure 1: Neutralino mass determination in the di-electron channel: Left: Di-electron invariant mass. Right: Di-electron energy. Both distributions show signal and all SM and SUSY backgrounds from full detector simulation after event selection.
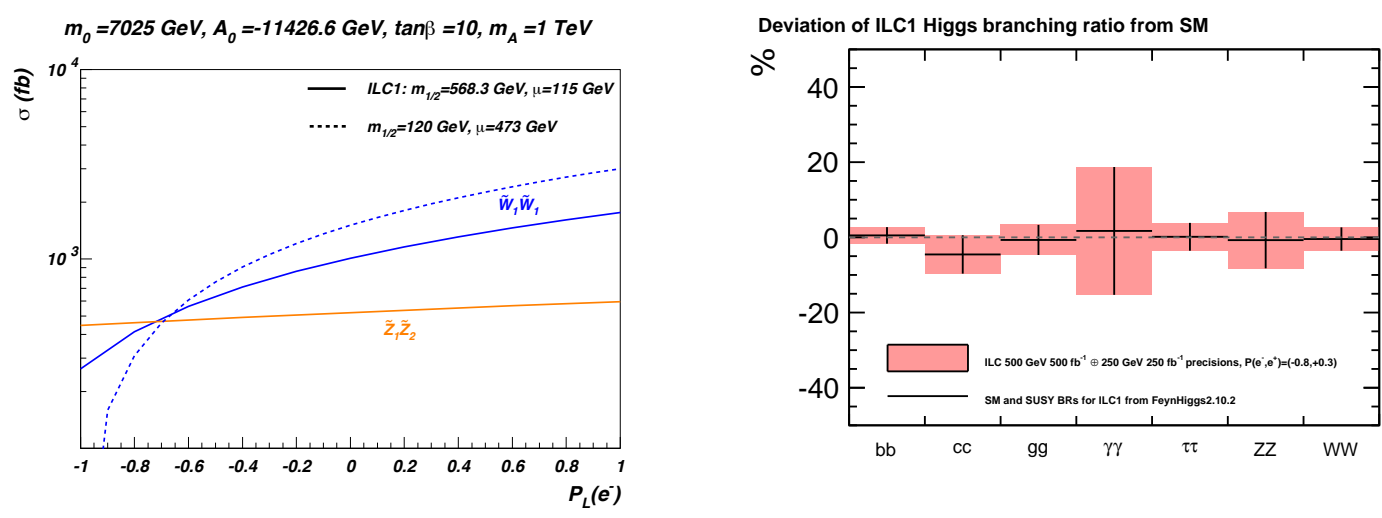

Figure 2: Left: Polarisation dependence of the production cross section for higgsino-like and wino-like charginos. Right: Expected Higgs branching ratio measurements for ILC1.

So far, we studied the leptonic decay of the neutral higgsino $e^{+} e^{-} \rightarrow \tilde{\chi}_{1}^{0} \tilde{\chi}_{2}^{0} \rightarrow \tilde{\chi}_{1}^{0} \tilde{\chi}_{1}^{0} e^{+} e^{-}\left(\mu^{+} \mu^{-}\right)$ and the semi-leptonic mode of charged higgsino production $e^{+} e^{-} \rightarrow \tilde{\chi}_{1}^{+} \tilde{\chi}_{1}^{-} \rightarrow \tilde{\chi}_{1}^{0} \tilde{\chi}_{1}^{0} q \bar{q}^{\prime} e v_{e}\left(\mu v_{\mu}\right)$. In both cases, the maximal invariant mass of the (virtual) intermediate vector boson (IVB) mediating the decay gives the mass splitting between the $\tilde{\chi}_{2}^{0}$ (or $\tilde{\chi}_{1}^{ \pm}$) and the LSP. The maximum energy of the same IVB gives the absolute masses via energy-momentum conservation, since at an $e^{+} e^{-}$ collider, the four-momentum of the initial state is known. Fig. 1 shows the corresponding distributions for the di-electron channel after event selection. Combining electron and muon channels, all masses can be extracted with precisions of about $1 \%$ from an initial ILC data set corresponding to an integrated luminosity of $500 \mathrm{fb}^{-1}$ at $\sqrt{s}=500 \mathrm{GeV}$.

The polarised beams of the ILC allow to measure the production cross sections for $P\left(e^{-}, e^{+}\right)=$ $(+80 \%,-30 \%)$ and $P\left(e^{-}, e^{+}\right)=(-80 \%,+30 \%)$ separately, which reveals the higgsino nature of the charginos, as illustrated in the left panel of Fig. 2. Precisions of $3 \%$ are predicted for each polarisation, again based on the initial ILC data set. 

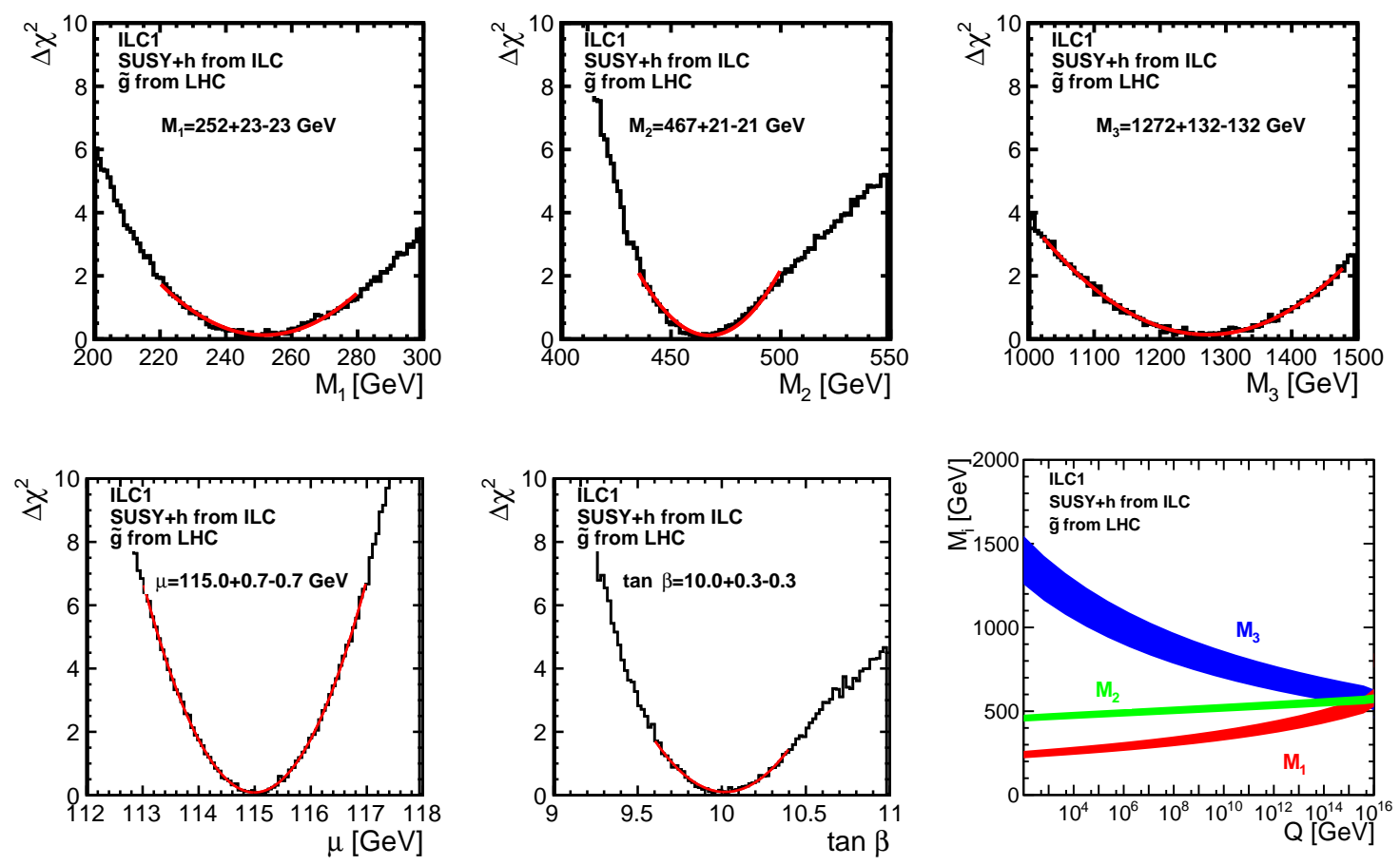

Figure 3: $\Delta \chi^{2}$ vs $\mu, \tan \beta, M_{1}, M_{2}, M_{3}$. The central values and $1 \sigma$ uncertainties are specified in the graphs. Lower right panel: running of the fitted $M_{1}, M_{2}$ and $M_{3}$ values to higher scales indicates mass unification at the GUT scale.

A further prediction of typical natural SUSY models is a nearly SM-like Higgs boson. This is illustrated for the ILC1 benchmark in the right panel of Fig. 2, where the shaded bands indicate the uncertainties of the model-independent coupling determination after the initial stage of the ILC [23]. In spite of their SM-likeness, the Higgs boson proporties will play an important role in the SUSY parameter determination.

The prospects for determining the weak-scale SUSY parameters from the measurements introduced in the previous section have been evaluated using FitTINo [24]. In addition to the ILC projections, we assumed a measurement of the gluino mass from the LHC with a precision of $10 \%$. However this assumption is not critical for any other parameter than $M_{3}$. Parameters which do not enter the electroweakino sector at tree-level have been fixed to their true values here. More general fits are in progress. Figure 3 shows $\Delta \chi^{2}$ vs each of the fitted parameters.

The resulting values and uncertainties for the weak-scale SUSY parameters can then be evolved to the GUT scale using 2-loop RGEs, here using SPHENo [25], as shown in the lower right panel of Fig. 3. This way, the hypothesis of gaugino mass unification at the GUT scale (here due to the underlying NUHM2 benchmark) could be tested based on the joint capabilities of LHC and ILC. Should the gluino be unobservable at the LHC, still the energy scale $Q$ for unification of $M_{1}$ and $M_{2}$ could be tested. Assuming also that $M_{3}$ unifies with them at the GUT scale, the gluino mass could then be predicted, giving important information for the design of the next generation hadron collider after the LHC. 


\section{Conclusions}

Motivated by the twin missives of simplicity and naturalness, then SUSY models with low $\mu$ and consequently light higgsinos of mass $\sim 100-300 \mathrm{GeV}$ are highly favored. Such light higgsinos would be easily visible at ILC with $\sqrt{s}>2 m_{\text {higgsino. }}$. Discovery of light higgsinos along with precision measurements of their properties would point to a natural origin for the EWSB sector and usher in a revolution in physics. This is indeed a powerful reason to promptly begin construction of ILC!

\section{References}

[1] H. P. Nilles, Phys. Rept. 110 (1984) 1.

[2] G. Aad et al. [ATLAS Collaboration], Phys. Lett. B 716 (2012) 1.

[3] S. Chatrchyan et al. [CMS Collaboration], Phys. Lett. B 716 (2012) 30.

[4] For a review, see e.g. M. S. Carena and H. E. Haber, Prog. Part. Nucl. Phys. 50 (2003) 63.

[5] The ATLAS collaboration, ATLAS-CONF-2015-067.

[6] G. Aad et al. [ATLAS Collaboration], Eur. Phys. J. C 75 (2015) no.10, 510 Erratum: [Eur. Phys. J. C 76 (2016) no.3, 153]; The ATLAS collaboration [ATLAS Collaboration], ATLAS-CONF-2016-077.

[7] H. Baer, V. Barger and A. Mustafayev, Phys. Rev. D 85 (2012) 075010; A. Arbey, M. Battaglia, A. Djouadi, F. Mahmoudi and J. Quevillon, Phys. Lett. B 708 (2012) 162.

[8] R. Barbieri and G. F. Giudice, Nucl. Phys. B 306 (1988) 63.

[9] S. Dimopoulos and G. F. Giudice, Phys. Lett. B 357 (1995) 573.

[10] J. Lykken and M. Spiropulu, Sci. Am. 310N5 (2014) 36.

[11] H. Baer, V. Barger, P. Huang, A. Mustafayev and X. Tata, Phys. Rev. Lett. 109, 161802 (2012).

[12] H. Baer, V. Barger, P. Huang, D. Mickelson, A. Mustafayev and X. Tata, Phys. Rev. D 87, 115028 (2013).

[13] H. Baer, V. Barger and D. Mickelson, Phys. Rev. D 88 (2013) no.9, 095013.

[14] H. Baer, V. Barger, D. Mickelson and M. Padeffke-Kirkland, Phys. Rev. D 89 (2014) no.11, 115019.

[15] A. Mustafayev and X. Tata, Indian J. Phys. 88 (2014) 991.

[16] H. Baer, V. Barger and M. Savoy, Phys. Rev. D 93 (2016) 3, 035016.

[17] K. J. Bae, H. Baer and E. J. Chun, Phys. Rev. D 89 (2014) no.3, 031701.

[18] H. Baer, V. Barger, D. Mickelson, A. Mustafayev and X. Tata, JHEP 1406 (2014) 172.

[19] R. Kitano and Y. Nomura, Phys. Rev. D 73 (2006) 095004; M. Papucci, J. T. Ruderman and A. Weiler, JHEP 1209 (2012) 035; C. Brust, A. Katz, S. Lawrence and R. Sundrum, JHEP 1203 (2012) 103.

[20] S. Agostinelli et al. [GEANT4 Collaboration], Nucl. Instrum. Meth. A 506 (2003) 250.

[21] W. Kilian, T. Ohl and J. Reuter, Eur. Phys. J. C 71 (2011) 1742 [arXiv:0708.4233 [hep-ph]].

[22] T. Sjostrand, S. Mrenna and P. Z. Skands, JHEP 0605 (2006) 026 [hep-ph/0603175].

[23] K. Fujii et al., arXiv:1506.05992 [hep-ex].

[24] P. Bechtle, K. Desch and P. Wienemann, Comput. Phys. Commun. 174 (2006) 47.

[25] W. Porod, Comput. Phys. Commun. 153 (2003) 275. 